\title{
NFKB inhibitors as a potential novel hypothesized treatment for psoriasis
}

\author{
Inibidores NFкB como um novo tratamento hipotético para psoríase
}

\author{
Nima Derakhshan'
}

'MD. Research Associate, Shiraz Nephro-Urology Research Center, Shiraz University of Medical

Sciences, Shiraz, Iran.
Psoriasis is a chronic, relapsing autoimmune skin disease that affects an estimated 1-3\% of the world's population. ${ }^{1}$ Identification of novel target pathways is necessary for treatment of this clinical enigma. Genetic, immunohistochemical and animal and human pharmacological studies support a possible role for nuclear factor kappa $\mathrm{B}(\mathrm{NF \kappa B})$ activation in the pathogenesis of psoriasis.

Among the genes considered to be important in psoriasis are the ones involved in apoptosis and cytokine production, via pathways such as those mediated by NFKB, which is a transcription factor that regulates hundreds of genes, including many involved in apoptosis., ${ }^{2,3}$ Thus, it may be considered to be a link between cell-mediated and keratinocyte-mediated mechanisms, which are believed to be the two different pathogenetic mechanisms of psoriasis. ${ }^{4}$

Immunohistochemical analysis on skin biopsies has revealed overexpression of NFKB in psoriatic epidermis, in comparison with normal epidermis. ${ }^{5}$ The Rel/NFKB transcription factors play a central role in numerous cellular processes, including keratinocyte proliferation and differentiation. Lizzul et al. designed a study in which a phosphorylation-specific antibody was used, and evaluated their results by means of immunohistochemistry. They proved that there was no expression of active NFKB in the normal epidermis of non-psoriatic skin, whereas a basal level of constitutive active phosphorylated NFKB/RelA was present in uninvolved epidermis in psoriasis patients. There was also significant upregulation of active phosphorylated NFKB/RelA in the epidermis from psoriatic plaques. ${ }^{6}$ These data suggest that activation of NFкB plays a significant role in the pathogenesis of psoriasis.

Several pharmacological studies have revealed that certain antipsoriatic drugs exert their action through inhibition of NFKB activation pathways. Acitretin has been found to decrease the expression of signal transducer and activator of transcription 1 (STAT1) and NFKB in nuclei of the human keratinocyte cell line (HaCaT), as determined by means of immunocytochemistry and Western blot. This suggests that the antipsoriatic effects of acitretin may be related to inhibition of nuclear translocations of STAT1 and NFKB. ${ }^{7}$ It has been shown that dimethylfumarate (DMF) inhibits NFKB translocation, which leads to its three possible antipsoriatic effects: (i) inhibition of pro-inflammatory cytokine production and adhesion molecule expression; (ii) inhibition of dendritic cell differentiation; and, at higher concentrations, (iii) induction of apoptosis. ${ }^{8}$ Rottlerin, which has been attributed with antipsoriatic properties, is not only an antioxidant but also a potent NFKB inhibitor. ${ }^{9}$ One potential mechanism of action for tumor necrosis factor-targeting agents in psoriasis is downregulation of NFKB transcriptional activity. Etanercept therapy in patients with psoriatic arthritis has been found to result in a significant decrease in NFKB, through inhibition of NFKB pathway. ${ }^{10}$

Animal studies have also suggested a possible role for NFKB signaling pathways in the pathogenesis of psoriasis. ${ }^{11,12}$ Marchetti et al. conducted a study that proved that increased expression of $\mathrm{NFKB}$ is an essential step in inducing a psoriasis-like inflammatory syndrome in mice. ${ }^{13}$ Functional blockade of NFKB by means of NFKB inhibitors in transgenic murine and human epidermis produced hyperplastic epithelium in vivo. Application of a pharmacological $\mathrm{NF \kappa B}$ inhibitor to intact skin was found to result in epidermal hyperplasia. In contrast, overexpression of active p50 and p $65 \mathrm{NF \kappa B}$ subunits in transgenic epithelium produced hypoplasia and growth inhibition. ${ }^{14}$ 
Studies on cytokine production pathways have also pointed towards a key role for NFKB activation in the pathogenesis of psoriasis. Transforming growth factor-alpha has been found to induce interleukin-6 in the human keratinocyte cell line (HaCaT) by means of transcriptional activation, possibly through NFKB activation. ${ }^{15}$ Insulin-like growth factor II (IGF-II), which has been shown to be higher in psoriatic patients, has been found to increase the interleukin-6 levels, possibly through NFKB activation. ${ }^{16}$

Emerging data gathered from several genetic, immunohistochemical and animal and human pharmacological studies support a possible role for NFKB activation pathways in the pathogenesis of psoriasis. This suggests that NFKB inhibitors may prove to be invaluable in treating psoriasis. The fact that this pathway is not activated in normal keratinocytes and only targets psoriatic skin lesions makes NFKB inhibitors a potential novel addition to the antipsoriatic weaponry.

\section{REFERENCES}

1. Namazi MR. Steroid sulfatase inhibitors as novel additions to the antipsoriatic armamentarium. Med Sci Monit. 2005;11(3):HY7-9.

2. Abdou AG, Hanout HM. Evaluation of survivin and NF-kappaB in psoriasis, an immunohistochemical study. J Cutan Pathol. 2008;35(5):445-51

3. Bowcock AM. The genetics of psoriasis and autoimmunity. Annu Rev Genomics Hum Genet. 2005;6:93-122.

4. Tsuruta D. NF-kappaB links keratinocytes and lymphocytes in the pathogenesis of psoriasis. Recent Pat Inflamm Allergy Drug Discov. 2009;3(1):40-8

5. Doger FK, Dikicioglu E, Ergin F et al. Nature of cell kinetics in psoriatic epidermis. J Cutan Pathol. 2007;34(3):257-63.

6. Lizzul PF, Aphale A, Malaviya R, et al. Differential expression of phosphorylated NF-kappaB/RelA in normal and psoriatic epidermis and downregulation of NF-kappaB in response to treatment with etanercept. J Invest Dermatol. 2005;124(6):1275-83.

7. Zhang $M$, Zhu L, Feng $Y$, et al. Effects of acitretin on proliferative inhibition and RANTES production of HaCaT cells. Arch Dermatol Res. 2008;300(10):575-81.

8. Mrowietz U, Asadullah K. Dimethylfumarate for psoriasis: more than a dietary curiosity. Trends Mol Med. 2005;11(1):43-8.

9. Maioli E, Valacchi G. Rottlerin: bases for a possible usage in psoriasis. Curr Drug Metab. 2010;11(5):425-30

10. Lories RJ, Derese I, Luyten FP, de Vlam K. Activation of nuclear factor kappa B and mitogen activated protein kinases in psoriatic arthritis before and after etanercept treatment. Clin Exp Rheumatol. 2008;26(1):96-102.

11. Wang H, Syrovets T, Kess D, et al. Targeting NF-kappa B with a natural triterpenoid alleviates skin inflammation in a mouse model of psoriasis. J Immunol. 2009;183(7):4755-63.
12. Schmidt KN, Podda M, Packer L, Baeuerle PA. Anti-psoriatic drug anthralin activates transcription factor NF-kappa B in murine keratinocytes. J Immunol. 1996;156(11):4514-9.

13. Marchetti S, Gamas P, Belhacène N, et al. The caspase-cleaved form of LYN mediates a psoriasis-like inflammatory syndrome in mice. EMBO J. 2009;28(16):2449-60.

14. Seitz CS, Lin Q, Deng H, Khavari PA. Alterations in NF-kappaB function in transgenic epithelial tissue demonstrate a growth inhibitory role for NF-kappaB. Proc Natl Acad Sci USA. 1998;95(5):2307-12.

15. Aragane $Y$, Yamada H, Schwarz A, et al. Transforming growth factoralpha induces interleukin-6 in the human keratinocyte cell line HaCaT mainly by transcriptional activation. J Invest Dermatol. 1996;106(6):1192-7.

16. Kwon YW, Jang ER, Lee YM, et al. Insulin-like growth factor II induces interleukin-6 expression via NFkappaB activation in psoriasis. Biochem Biophys Res Commun. 2000;278(2):312-7.

Sources of funding: None

Conflict of interest: None

Date of first submission: March 19, 2011

Last received: June 16, 2011

Accepted: June 16, 2011

\section{Address for correspondence:}

Nima Derakhshan

Shiraz Nephro-Urology Research Center

Shiraz University of Medical Sciences,

Shiraz, Iran

Tel.: (+98) 9177161290

E-mail: nima_med83@yahoo.com 